\title{
MHD instability in differentially-rotating cylindric flows
}

\begin{abstract}
G. Rüdiger and Y. Zhang
Astrophysikalisches Institut Potsdam, An der Sternwarte 16, 14482 Potsdam, Germany

Received 28 March 2001 / Accepted 24 August 2001

Abstract. The possibility that the magnetic shear-flow instability (MRI, also Balbus-Hawley instability) might give rise to turbulence in a cylindrical Couette flow is investigated through numerical simulations. The study is linear and the fluid is assumed to be incompressible and differentially rotating with the rotation law $\Omega=a+b / R^{2}$. The model is fully global in all three spatial directions with boundaries on each side; finite diffusivities are also imposed. The computations are carried out for several values of the azimuthal wavenumber $m$ of the perturbations in order to analyze whether or not non-axisymmetric modes are preferred, which in a nonlinear extension of the study finally might lead to a dynamo-generated magnetic field. For magnetic Prandtl number of order unity we find that with a magnetic field the instability is generally easier to excite than without a magnetic field. The critical Reynolds number for $\mathrm{Pm}=1$ is of the order of 50, independent of whether or not the nonmagnetic flow is stable. We find that i) the magnetic field strongly reduces the number of Taylor vortices, ii) the angular momentum is transported outwards and iii) for finite cylinders a net dynamo-alpha effect results which is negative (positive) for the upper (lower) part of the cylinder. For magnetic Prandtl number smaller than unity the critical Reynolds number appears to scale with $\mathrm{Pm}^{-0.65}$. If this was true even for very small magnetic Prandtl numbers (e.g. for $10^{-5}$, the magnetic Prandtl number of liquid sodium) the critical Reynolds number should reach the value of $10^{5}$ which, however, is also characteristic of the nonlinear finite-amplitude hydrodynamic Taylor-Couette turbulence - so that we have to expect the simultaneous existence of both sorts of instabilities in related experiments. Similar phenomena are also discussed for cold accretion disks with their basically small magnetic Prandtl numbers.
\end{abstract}

Key words. magnetohydrodynamics - accretion, accretion disks - turbulence

\section{Introduction}

In recent years the generation of turbulence in accretion disks has been thought to be based on the magnetorotational instability (MRI, also Balbus-Hawley instability) in which differential rotation with outward-decreasing rotation rate becomes unstable under the influence of a given magnetic field (Velikhov 1959; Chandrasekhar 1961). After the initial publications (see Balbus \& Hawley 1991) several other papers have studied the nonlinear evolution of the instability (Hawley \& Balbus 1991; Brandenburg et al. 1995; Hawley et al. 1995; Matsumoto \& Tajima 1995). Using global investigations, the importance of boundaries has been noted, in particular with respect to the influence of the excitation of nonaxisymmetric modes, which after Cowling's theorem, are necessary for the self-excitation of magnetic fields (Curry et al. 1994; Curry \& Pudritz 1995, 1996; Kitchatinov \& Rüdiger 1997; Kitchatinov \& Mazur 1997; Rüdiger et al. 1999). All these studies were, however, linear; a fully global nonlinear approach just beginning (e.g. Drecker et al. 2000; Hawley 2000; Arlt \& Rüdiger 2001).

Send offprint requests to: G. Rüdiger,

e-mail: gruediger@aip.de
On the other hand, Dubrulle (1993), Richard \& Zahn (1999) and Duschl et al. (2000) focus on the existence of instabilities in hydrodynamics, i.e. nonmagnetic TaylorCouette flow as a configuration between two rotating cylinders (see also Koschmieder 1993). Here we have to distinguish between two cases. For steep rotation laws, i.e. for sufficiently slow rotation of the outer cylinder and sufficiently rapid rotation of the inner cylinder, there is indeed a linear nonmagnetic instability, which is, however, not interesting for astrophysical applications such as accretion disks. The instability is due to the violation of the Rayleigh stability criterion, $\mathrm{d}\left(R^{2} \Omega\right) / \mathrm{d} R>0$, which for Kepler rotation is always fulfilled.

One can ask, however, about the appearance of nonlinear instabilities due to finite-amplitude disturbances. They occur indeed in Taylor-Couette flow experiments, but for Reynolds numbers exceeding those of the linear instability by several orders of magnitudes. For a rather wide gap between the two cylinders, the data discussed by Richard \& Zahn (1999) lead to a Reynolds number for finite-amplitude instability of about $2 \times 10^{5}$ (see below).

However, it is true that examples for nonlinearly unstable Kepler flows are not known so far. Numerical experiments with finite disturbances have demonstrated that 
turbulent structures in Kepler flows always disappeared after a few rotation periods when the perturbations were switched off. We did not find an effective perturbation of the basic Kepler flow that resulted in long-lived turbulent disturbances (Arlt 2000; Rüdiger \& Drecker 2001). However, it is also true that the Reynolds numbers of the numerical simulations which can be realized with current computers are too small compared to the Reynolds numbers of real accretion disks. Hence, in particular for cold protoplanetary disks with their very low electrical conductivity, the possible existence of hydrodynamic finiteamplitude instabilities as the source of the necessary angular momentum transport cannot finally be ruled out.

In the present paper we shall demonstrate that for MHD Taylor-Couette flow experiments with electrically conducting fluids in the presence of an external field there are always linear instabilities for rather moderate Reynolds numbers, independent of whether or not the hydrodynamic Taylor-Couette flow is unstable. We are able to compute the MRI for magnetic Prandtl numbers of $0.01 \leq \mathrm{Pm} \leq 1$. However, both for cold accretion disks and liquid metals like sodium or gallium, the magnetic Prandtl numbers are much smaller. For such small Prandtl numbers of order (say) $10^{-5}$ an extrapolation is needed leading to a Reynolds number of about $10^{5}$. So we have the interesting situation in which for natural electricallyconducting fluids the critical Reynolds number for linear MHD-turbulence approaches the critical Reynolds number for nonlinear hydrodynamic turbulence. If this is true then complications for an experimental realization of the MRI are predicted - and possibly also complications for the theory of protoplanetary disks.

Here we investigate the instability in a numerical model for a Taylor-Couette flow in which a magnetized incompressible fluid is contained between two rotating finite cylinders (Fig. 1). In the absence of viscosity, the class of such permissible flows is very wide. However, if viscosity is present the class becomes restricted: in fact, in the absence of any transverse pressure gradient, the most general form of $\Omega$ which is allowed is

$\Omega(R)=a+\frac{b}{R^{2}}$,

where $a$ and $b$ are two constants which are related to the angular velocities $\Omega_{\text {in }}$ and $\Omega_{\text {out }}$ with which the inner and the outer cylinders are rotating. If $R_{\text {in }}$ and $R_{\text {out }}\left(>R_{\text {in }}\right)$ are the radii of the two cylinders then

$\Omega_{\mathrm{in}}=a+b / R_{\mathrm{in}}^{2}, \quad \Omega_{\mathrm{out}}=a+b / R_{\mathrm{out}}^{2}$.

Solving for $a$ and $b$ in terms of $\Omega_{\text {in }}$ and $\Omega_{\text {out }}$ we have

$a=\Omega_{\text {in }} \frac{\hat{\mu}-\hat{\eta}^{2}}{1-\hat{\eta}^{2}}, \quad b=\Omega_{\text {in }} \frac{R_{\text {in }}^{2}(1-\hat{\mu})}{1-\hat{\eta}^{2}}$,

where $\hat{\mu}=\Omega_{\text {out }} / \Omega_{\text {in }}$ and $\hat{\eta}=R_{\text {in }} / R_{\text {out }}$.

Flow and field perturbations are developed after the azimuthal Fourier modes $\exp (\mathrm{i} m \phi)$. Although usually the flow and field quantities are then complex numbers, only their real parts have a physical meaning.

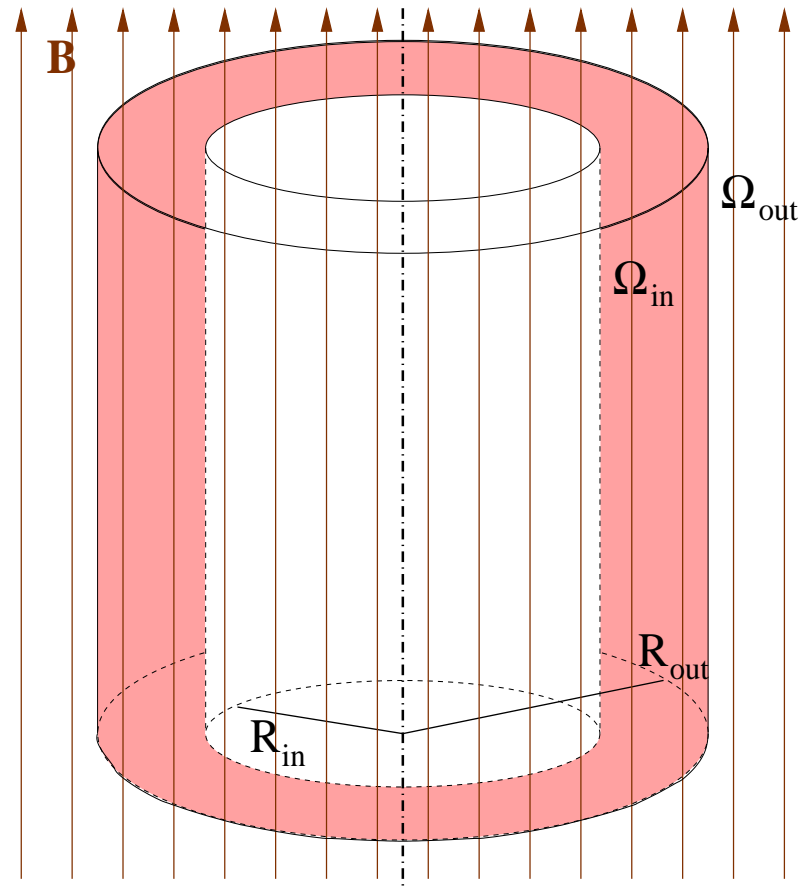

Fig. 1. Geometry of the cylindric Taylor-Couette flow.

\section{Equations and model}

We solve the incompressible, dissipative, linearized MHD equations numerically in cylindrical coordinates for a global model. The basic rotation law is given in (1) and the rotating fluid may be threaded by a vertical magnetic field

$\boldsymbol{B}_{0}=B_{0} e_{z}$

(cf. Stone \& Norman 1994). Extra radially-dependent azimuthal fields are also possible in this approximation (Curry \& Pudritz 1995; Ogilvie \& Pringle 1996; Terquem \& Papaloizou 1996; Papaloizou \& Terquem 1997). By linearizing around the equilibrium state and using dimensionless quantities, our equations read

$$
\frac{\partial \boldsymbol{u}}{\partial t}=C_{\Omega}(-\mathcal{A}-\nabla p)+\left(\frac{\mathrm{Ha}^{2} \mathrm{Pm}}{C_{\Omega}}\right) \mathcal{L}+\operatorname{Pm} \Delta \boldsymbol{u}
$$

$$
\Delta p=\boldsymbol{\nabla} \cdot\left(-\mathcal{A}+\left(\frac{\mathrm{Ha}^{2} \mathrm{Pm}}{C_{\Omega}^{2}}\right) \mathcal{L}+\frac{\mathrm{Pm}}{C_{\Omega}} \Delta \boldsymbol{u}\right)
$$

$\frac{\partial \boldsymbol{B}}{\partial t}=\operatorname{curl}\left(C_{\Omega}(\boldsymbol{\mathcal { R }}+\mathcal{E})-\operatorname{curl} \boldsymbol{B}\right)$

where $p$ is the pressure divided by the density, $\boldsymbol{u}$ the velocity perturbation and $\boldsymbol{B}$ the magnetic perturbation. The normalizations are the same as in Rüdiger et al. (1999).

In the equations $\mathcal{A}$ and $\mathcal{L}$ are the linearized advection term and Lorentz force given by

$\mathcal{A}=\frac{\Omega}{\Omega_{\text {in }}}\left(\begin{array}{c}\mathrm{i} m u_{R}-2 u_{\phi} \\ \mathrm{i} m u_{\phi}+2 \frac{a}{\Omega} u_{R} \\ \mathrm{i} m u_{Z}\end{array}\right)$ 

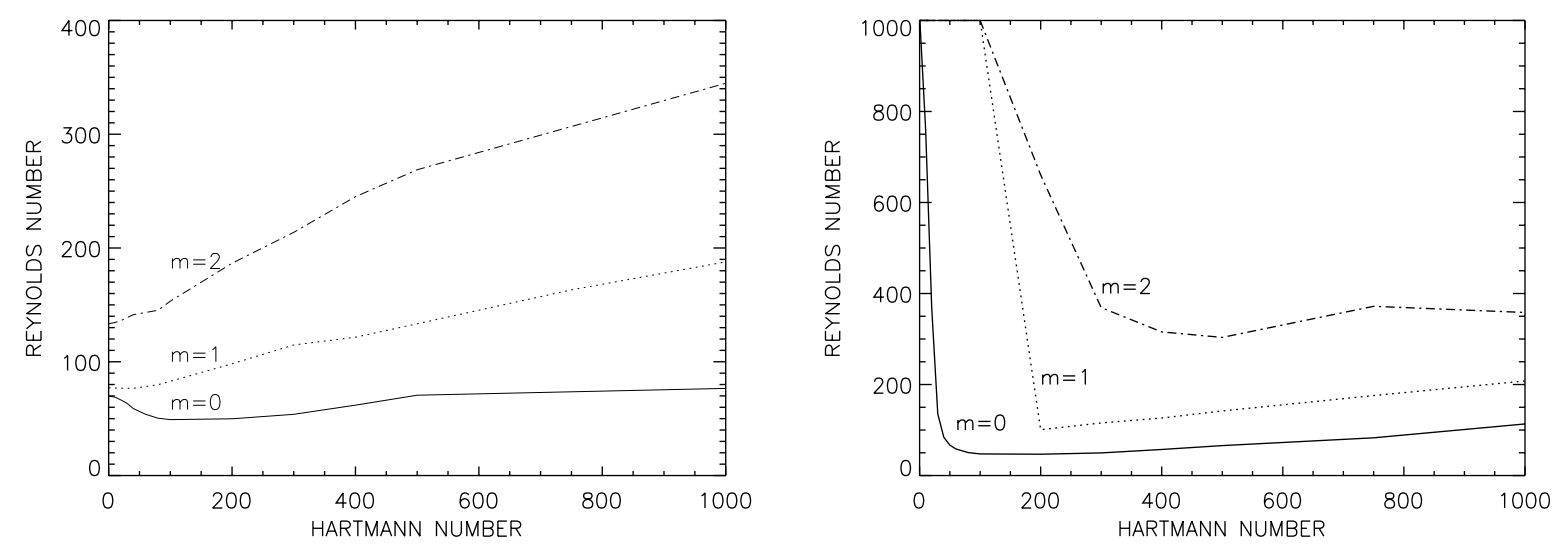

Fig. 2. Neutral-stability lines for various azimuthal wave numbers $(m=0,1,2)$ and rigid boundary conditions. The outer cylinder is at rest (left, hydrodynamic instability exists) or it rotates with $33 \%$ of the rotation rate of the inner cylinder (right, hydrodynamic instability does not exist). $\mathrm{Pm}=1, \hat{\eta}=0.5$

and

$\mathcal{L}=\left(\begin{array}{c}\frac{\partial B_{R}}{\partial z}-\frac{\partial B_{Z}}{\partial r} \\ -\frac{\mathrm{i} m}{r} B_{Z}+\frac{\partial B_{\phi}}{\partial z} \\ 0\end{array}\right)$,

while

$\mathcal{R}=r \frac{\Omega}{\Omega_{\text {in }}}\left(\begin{array}{c}B_{Z} \\ 0 \\ -B_{R}\end{array}\right)$

and

$\mathcal{E}=\left(\begin{array}{c}u_{\phi} \\ -u_{R} \\ 0\end{array}\right)$

are the linearized electromotive force induced by the basic differential rotation and the flow perturbations. In the equations above $r$ denotes the dimensionless radius $r=R / H$,

$\mathrm{Ha}=\frac{B_{0} H}{\sqrt{\mu_{0} \rho \nu \eta}}$

is the Hartmann number $(\eta, \rho$ and $\nu$ being the magnetic diffusivity, density and viscosity);

$C_{\Omega}=\frac{\Omega_{\text {in }} H^{2}}{\eta}$

is the shear-flow parameter ("dynamo number") and Pm is the magnetic Prandtl number, $\mathrm{Pm}=\nu / \eta$. The quantity $H$ is the half-thickness of the cylinder which is also the unit length for the non-dimensionalization. The unit time is the magnetic diffusion time $\tau=H^{2} / \eta$, velocities are normalized with $H \Omega_{\text {in }}$. A time-stepping code is used with a global energy-quenching procedure of the form $C_{\Omega}=C_{\Omega}^{(0)} /(1+E)$ to find the lowest excitation regime, where $E$ is the total energy of the system (see Elstner et al. 1990). In our computations we also use the Reynolds number,

$\mathrm{Re}=\frac{\Omega_{\text {in }} R_{\text {in }}\left(R_{\text {out }}-R_{\text {in }}\right)}{\nu}$, so finally we get

$\operatorname{Re}=\frac{C_{\Omega} \hat{\eta}(1-\hat{\eta})}{\operatorname{Pm}^{2}}$

with $\Gamma=H / R_{\text {out }}$.

With our definitions the nonlinear instability described by Richard \& Zahn (1999) - using experimental data of Wendt (1933) and Taylor (1936) - starts for

$\operatorname{Re} \gtrsim 5 \times 10^{6} \frac{\hat{\eta}(1-\hat{\eta})^{2}}{(1+\hat{\eta})^{3}(1-\hat{\mu})}$,

a number which is about $2 \times 10^{5}$ for $\hat{\mu}=0$ and $\hat{\eta}=0.5$ used in our computations below.

A staggered mesh of 81 by 81 grid points was used to solve the system. In Eqs. (5)-(7) Ha, $C_{\Omega}$ and Pm are free parameters. We solved the equations in order to get a representation of the curves of marginal stability in the Ha- $C_{\Omega}$ plane.

We impose rigid boundary conditions for the velocity field, both taken on all boundary layers and set pseudovacuum boundary conditions on the magnetic field, i.e. the components of the magnetic flux tangential to the surface are vanishing. This choice also is used for simplicity in imposing the conditions for the flow, since by using a staggered mesh the grid-points for the velocity are not defined directly on the boundaries. It also ensures the necessary vanishing of the Poynting vector in the normal direction (cf. Ruzmaikin et al. 1988). It is generally accepted that the MRI is influenced by the presence of boundaries (Curry \& Pudritz 1995). It is thus of much importance to find out how the various boundary conditions influence (say) the symmetry of the solutions with respect to the rotation axis or with respect to the equator. Local simulations for closed boxes cannot answer questions that are of observational significance.

\section{Results}

\subsection{The neutral-stability lines}

The neutral-stability lines for $m<3$ in the Re-Ha plane for $\mathrm{Pm}=1$ and various $\hat{\mu}$ are given in Fig. 2. The curves 
for $\hat{\mu}=0$ have an intersection with the Re-axis; for $m=0$ and $\mathrm{Ha}=0$ we find $\mathrm{Re}=69.75$. This case is well-known for the nonmagnetic flow, the exact solution is $\mathrm{Re}=68.3$ (Chandrasekhar 1961). Both values are close to each other and our numerical simulations are thus consistent with the experiment. There is also a minimum at $\mathrm{Re}=49.15$ for the line with $m=0$. We conclude that with magnetic field the instability is easier to excite than without a magnetic field. For stronger magnetic fields the critical Reynolds number again increases so that - as known - the instability is suppressed by high magnetic field amplitudes.

In Fig. 2 flat rotation laws are also considered, where no hydrodynamical instability exists $(\hat{\mu}=0.33)$. The eigenvalue for $\mathrm{Ha}=0$ moved to infinity. With a magnetic field, however, the minimum of the Reynolds number is barely changed $(\mathrm{Re}=46.9)$ compared to the former case. It can be stressed that for Hartmann numbers of order 100 there is always a critical Reynolds number of order 50 above which we have instability - independent of whether or not hydrodynamic instability exists.

The results might be applied to two different cases. The first one is the solar tachocline with its values of $\nu \simeq$ $10 \mathrm{~cm}^{2} / \mathrm{s}$ and $\eta \simeq 10^{3} \mathrm{~cm}^{2} / \mathrm{s}\left(\mathrm{Pm}=10^{-2}\right.$, see Rüdiger \& Kitchatinov 1996). The Reynolds numbers are much higher than $10^{2}$. It follows that $B_{0}=10^{-9} \mathrm{Ha} / \epsilon$ Gauss for the characteristic magnetic field strength with $\epsilon=H / R$. With $\mathrm{Ha} \simeq 10^{2}$ and $\epsilon \approx 0.1$ one finds $B_{0} \simeq 1 \mu$ Gauss.

On the other hand, an experiment with liquid sodium with $\mathrm{Pm}=10^{-5}\left(\nu=10^{-2} \mathrm{~cm}^{2} / \mathrm{s}, \eta=10^{3} \mathrm{~cm}^{2} / \mathrm{s}\right)$ leads to $B_{0} \approx \mathrm{Ha} / 10$ in Gauss for $H \simeq 100 \mathrm{~cm}$, i.e. $B_{0} \simeq 10$ Gauss for $\mathrm{Ha} \simeq 100$. With $R_{\text {in }}$ and $\Delta R$ of $10 \mathrm{~cm}$ each, it is $\mathrm{Re} \simeq 10^{5} f$ with the rotation frequency $f$, so that it should not be a problem to reach critical Reynolds numbers of the order of $10^{5 \ldots 6}$.

\subsection{The eigenfunctions}

It is known that the vertical cell size for the linear nonmagnetic Taylor-Couette flow is typically the same as it is in the radial direction - we therefore find 10 cells in Fig. 3 for $\mathrm{Ha}=0$. With a magnetic field only 5 cells exist with antisymmetry with respect to the equator (Fig. 4). Obviously, the value of the vertical wave number strongly decreases for increasing magnetic field (Chandrasekhar 1961).

In Fig. 4 the induced toroidal magnetic field is also shown. It is an equatorially symmetric (" $S$ ") field. Correspondingly, the associated zonal kinetic flow has an antisymmetric structure with respect to the equator. The induced fields and flows here are concentrated at the boundary close to the inner cylinder.

\subsection{Viscosity-alpha and dynamo-alpha}

Although our study is linear, it is worth analyzing the angular momentum transport of the instability. The effective viscosity responsible for the angular momentum transport is generally parameterized by the quantity $\alpha_{\mathrm{SS}}$

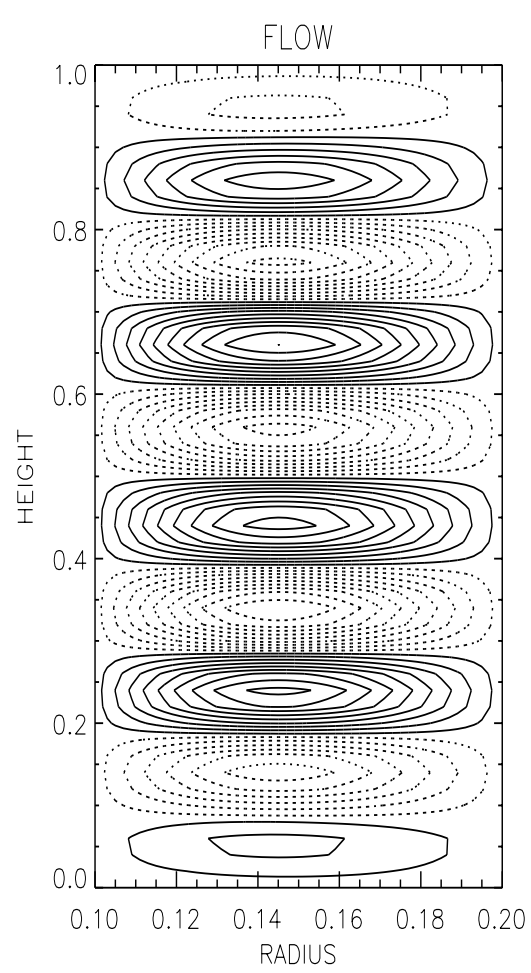

Fig. 3. The pattern for the the azimuthal flow without magnetic field $(\mathrm{Ha}=0)$ but for a resting outer cylinder $(\hat{\mu}=0)$. $\hat{\eta}=0.5, \mathrm{Pm}=1, m=0$.

(Shakura \& Sunyaev 1973) defined in terms of the Reynolds and Maxwell stress tensor components by

$\left\langle u_{R} u_{\phi}-\frac{B_{R} B_{\phi}}{\mu_{0} \rho}\right\rangle=-\nu_{\mathrm{T}} R \frac{\partial \Omega}{\partial R}$

with the normalization

$\nu_{\mathrm{T}}=\alpha_{\mathrm{SS}} H^{2} \Omega$

where $\nu_{\mathrm{T}}$ is the eddy viscosity and $\alpha_{\mathrm{SS}}$ is the viscosityalpha. As we know, the $\alpha_{\mathrm{SS}}$ is not trivially positive, there are several nonmagnetic simulations with negative correlations $\left\langle u_{R} u_{\phi}\right\rangle$ (Ryu \& Goodman 1992; Cabot \& Pollack 1992; Kley et al. 1993; Stone \& Balbus 1996).

In dimensionless units

$\alpha_{\mathrm{SS}} \propto\left\langle u_{R} u_{\phi}-\frac{\mathrm{Ha}^{2} \mathrm{Pm}}{C_{\Omega}^{2}} B_{R} B_{\phi}\right\rangle$.

In our linear approach the amplitudes of the quantities are unknown as they are free to a common positive or negative factor. However, the sign can be computed for the quadratic correlation terms and/or ratios of fluctuating quantities.

Here the turbulent electromotive force $\mathcal{E}=\left\langle\boldsymbol{u}^{\prime} \times \boldsymbol{B}^{\prime}\right\rangle$ is considered which is due to the external field, i.e. $\mathcal{E}_{z}=$ $\alpha_{z z} B_{z}$. Apart from positive factors we find

$\alpha_{z z} \propto\left\langle u_{R} B_{\phi}-u_{\phi} B_{R}\right\rangle$,

where the averaging procedure must distinguish between upper and lower parts of the cylinder. The alpha-tensor 

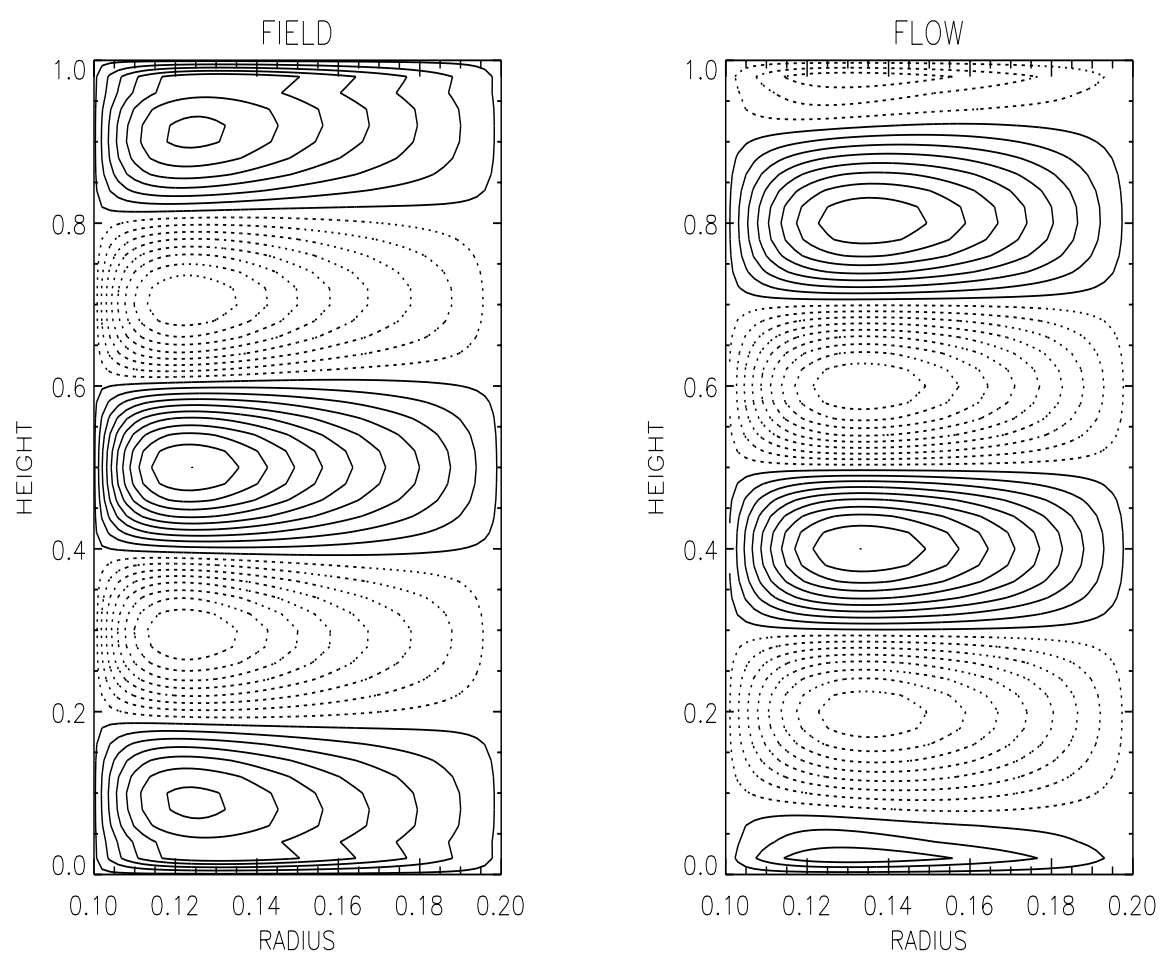

Fig. 4. The eigenfunctions for the toroidal magnetic field (left) and the azimuthal flow (right) in the gap between the cylinders. $\mathrm{Ha}=200, \mathrm{Pm}=0.1, m=0$

is a pseudo-tensor. Hence, for a rotating container it can only be formed by the pseudo-scalar $\boldsymbol{g} \cdot \boldsymbol{\Omega}$ if $\boldsymbol{g}$ is a preferred direction derived from the geometry. For the considered container the only interesting preferred direction is perpendicular to the top and bottom boundary surfaces ${ }^{1}$. The alpha-effect is thus antisymmetric with respect to the midplane between the top and bottom boundaries. So the remaining question is whether it is positive in the upper part and negative in the lower part or v.v. The results are presented in Figs. 6 and 7. Figure 6 for a prescribed radius we display the vertical profile of $\hat{\alpha} \equiv u_{R} B_{\phi}-u_{\phi} B_{R}$ for $m=0$ which has to be averaged to find the alpha-effect. The result is quite typical for the nature of this effect. The flow pattern forms cells. The lower part of a cell has a negative (positive) $\hat{\alpha}$ in the upper (lower) part of the cylinder. The upper part of the cell is opposite. Due to the influence of the upper and lower boundaries, however, the $\hat{\alpha}$ of the lower part of a cell dominates the $\hat{\alpha}$ of the upper part of a cell. The total (vertical) alpha for each cell is thus negative (positive) for the upper (lower) part of the cylinder. Figure 7 gives the resulting alpha-effect after averaging $\hat{\alpha}$ about the entire upper part of the cylinder or the lower part, resp. Note that our computations lead to a negative (positive) total alpha-effect for the upper (lower) part of the cylinder. This result seems to be characteristic for the MRI (see Brandenburg 1998).

Obviously, for an infinite cylinder (or a model with periodic boundary conditions in $z$ ) there is no vertical

\footnotetext{
1 The radial direction defined by the curvature of the cylinders is perpendicular to $\boldsymbol{\Omega}$ and does not play a role.
}

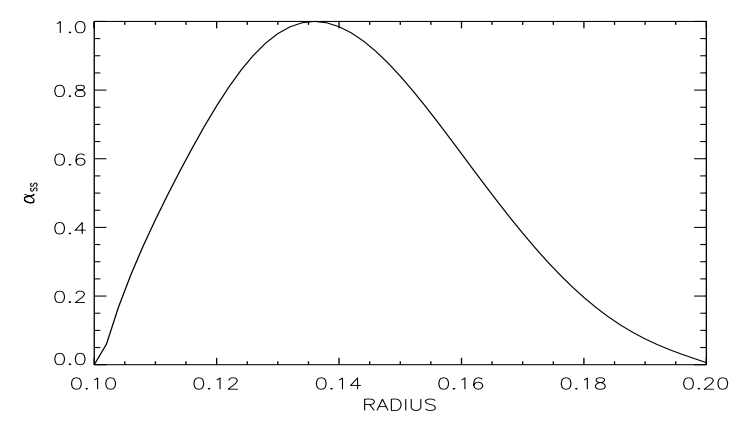

Fig. 5. The viscosity-alpha, $\alpha_{\mathrm{SS}}$, is always positive, i.e. the angular momentum transport is outwards, even for the axisymmetric cells $(m=0)$. Ha $=200, \hat{\mu}=0.33, \hat{\eta}=0.5$.

preferred direction and, therefore, no alpha-effect. Our linear theory, of course, gives no results about the amplitude of this effect and, therefore, also no findings about a possible dynamo action of the cylindric Taylor-Couette flow. The latter can only be discussed in the frame of nonlinear simulations.

\section{The magnetic Prandtl number dependence}

In order to approach real plasmas we have to switch to magnetic Prandtl number smaller than unity. The solar gas beneath the convection zone approaches Prandtl numbers of order $10^{-2}$ while liquid sodium, due to its small viscosity of $10^{-5}$, is even more extreme. 


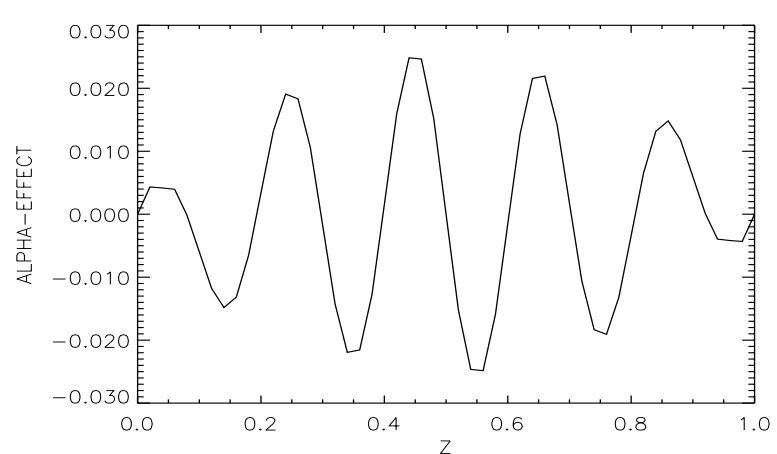

Fig. 6. The nonlinear quantity $\hat{\alpha}$ non-averaged as $z$-profile for $m=0$ and at a given radius. $\mathrm{Pm}=0.1, \mathrm{Ha}=200, \hat{\mu}=0.33$, $\hat{\eta}=0.5$.

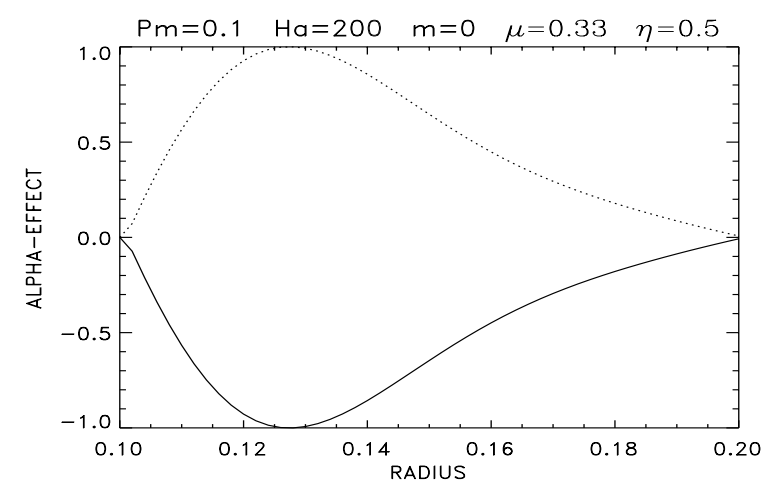

Fig. 7. The (vertical) dynamo-alpha is negative in the upper hemisphere (solid) and positive in the lower hemisphere (dashed).

Simulations with $\mathrm{Pm}=0.1$ for a flat basic rotation law are given in detail in Fig. 8. The lowest eigenvalue is now about 171 . Some computations with $\mathrm{Pm}=0.01$ are also done. Figure 9 suggests a power-law behaviour with

$\operatorname{Re}_{\text {crit }} \propto \operatorname{Pm}^{-0.65}$,

which we have only justified, however, down to $\mathrm{Pm}=0.01$. If it was also true for magnetic Prandtl numbers of order $10^{-5}$, the critical Reynolds number would be $9 \times 10^{4}$. This value is very close to the Reynolds number which we know from (16) for nonlinear, nonmagnetic instability. It might be the case that in the limit of very small magnetic Reynolds number, i.e. for very cold accretion disks, we have the realization of a mixed instability: linear magneto-rotational instability and nonlinear hydromagnetic Taylor-Couette flow turbulence.

\section{Discussion}

In the present paper differentially rotating cylindric flows are considered which are linearly stable in the nonmagnetic regime but which are linearly unstable in the hydromagnetic regime. The computations are motivated both by discussions of nonlinear hydrodynamic instabilities for

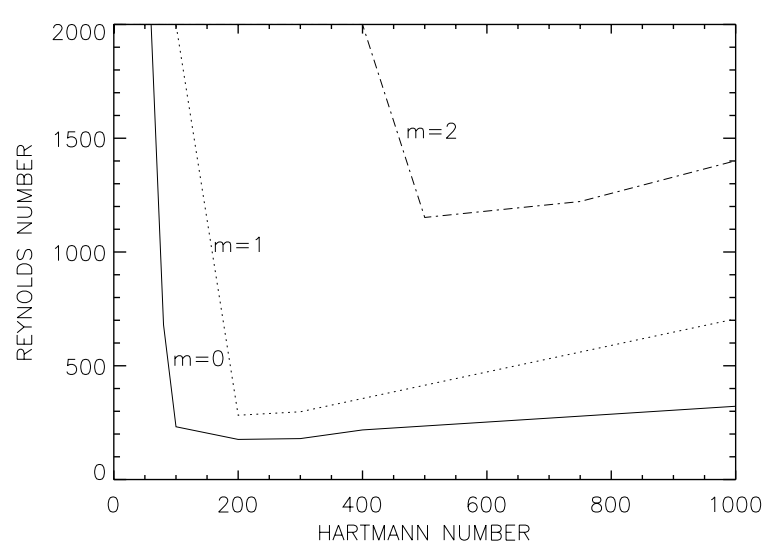

Fig. 8. Neutral-stability lines for various azimuthal wave numbers $(m=0,1,2)$ and rigid boundary conditions with $\mathrm{Pm}=$ 0.1 . Hydrodynamic instability does not exist. The minimum Reynolds number is about $176 . \hat{\mu}=0.33, \hat{\eta}=0.5$.

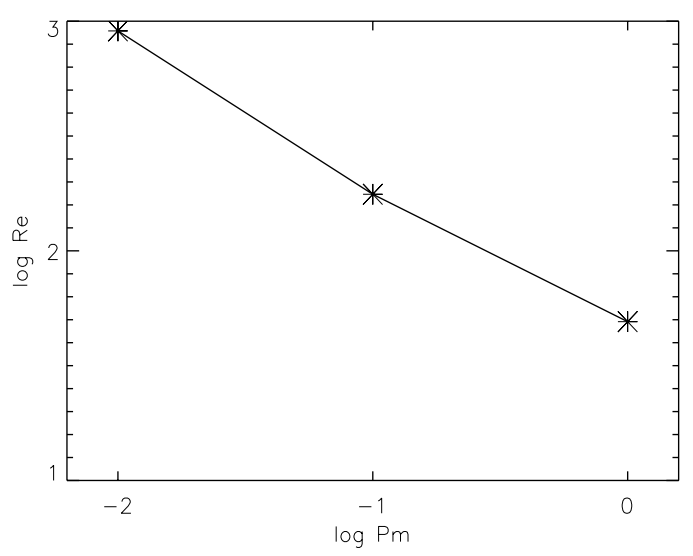

Fig. 9. Critical Reynolds numbers for various magnetic Prandtl numbers. The Reynolds number increases for decreasing Prandtl number. $m=0$.

high Reynolds numbers as a possible explanation for turbulence in protoplanetary disks (cf. Richard \& Zahn 1999) as well as the theoretical preparation of technical TaylorCouette flow experiments of the magnetorotational instability for small magnetic Prandtl numbers including new dynamo experiments.

It is shown that for both flat or steep rotation laws the MHD Taylor-Couette flow is always linearly unstable. In contrast to the nonmagnetic regime the critical Reynolds number for instability does not strongly depend on the rotation rate of the outer cylinder, provided it rotates slower than the inner one. The dependence of the critical Reynolds number on the magnetic Prandtl number, however, is much stronger, see Eq. (20). It is, however, probably not too strong to question experimental work with liquid sodium with its low magnetic Prandtl number of order $10^{-5}$. For such small magnetic Prandtl numbers the estimated Reynolds number is of the order of $10^{5}$, which is already known from experiments concerning the nonlinear instability of nonmagnetic Taylor-Couette flow. 
So we have the puzzling situation that the Reynolds number for two very different types of instability could be very similar, i.e. for both nonlinear hydrodynamic instability and linear magnetohydrodynamic instability. If true, such a result will also have implications for the simulations of protoplanetary disks, which due to the low temperatures also have rather low magnetic Prandtl numbers.

It should be noted that our code only works for magnetic Prandtl numbers down to $10^{-2}$. The critical Reynolds numbers for lower Prandtl numbers are here only extrapolated. A new code is needed to find out the eigenvalues for very small magnetic Prandtl numbers.

Of particular interest also for technical realizations should be the existence of a turbulent electromotive force $\mathcal{E}=\left\langle\boldsymbol{u}^{\prime} \times \boldsymbol{B}^{\prime}\right\rangle$. Although its magnitude here must remain open as the theory is linear, we can find the sign of all the bilinear expressions. The EMF divided by the uniform external field yields the dynamo-alpha for the container. As only a vertical magnetic field is applied here, we can only fix the $z z$-component of the alpha-tensor. This $\alpha_{z z}$ proves to be negative (positive) in the upper (lower) part of the cylinder. It only exists due to the bounding of the container in the vertical direction. One can try to understand this finding using the following consideration. In order to construct the pseudo-tensor $\alpha$ one needs a scalar $\boldsymbol{g} \cdot \boldsymbol{\Omega}$ with a characteristic direction $\boldsymbol{g}$. In a vertically infinite cylinder the only characteristic direction $\boldsymbol{g}$ is the radial one, but then it is $\boldsymbol{g} \cdot \boldsymbol{\Omega}=0$. Only for cylinders which are bounded in the vertical, $\boldsymbol{g} \| \boldsymbol{\Omega}$ acts as a characteristic direction leading to a finite alpha-effect with the required antisymmetry with respect to the equator. Thus we can confirm the assertion by Hawley et al. (1996) that 'more than shear and turbulence are required to produce dynamo amplification of magnetic fields'.

Acknowledgements. The anonymous referee is acknowledged for valuable suggestions improving the philosophy of the presented computations. Axel Brandenburg (Kopenhagen) is acknowledged for a critical reading of the manuscript.

\section{References}

Arlt, R. 2000, Thesis, University of Potsdam

Balbus, S. A., \& Hawley, J. F. 1991, ApJ, 376, 214

Brandenburg, A., Nordlund, A., Stein, R. F., \& Torkelsson, U. 1995, ApJ, 446, 741
Brandenburg, A. 1998, Disc turbulence and viscosity, in Theory of black hole accretion disks, ed. M. A. Abramowicz, G. Björnsson, \& J. E. Pringle (Cambridge University Press, Cambridge), 61

Cabot, W., \& Pollack, J. B. 1992, Geophys. Astrophys. Fluid Dyn., 64, 97

Chandrasekhar, S. 1961, Hydrodynamic and Hydromagnetic Stability (Clarendon, Oxford)

Curry, C., Pudritz, R. E., \& Sutherland, P. G. 1994, ApJ, 434, 206

Curry, C., \& Pudritz, R. E. 1995, ApJ, 453, 697

Curry, C., \& Pudritz, R. E. 1996, MNRAS, 281, 119

Drecker, A., Rüdiger, G., \& Hollerbach, R. 2000, MNRAS, 317, 45

Dubrulle, B. 1993, Icarus, 106, 59

Duschl, W. J., Strittmatter, P. A., \& Biermann, P. L. 2000, A\&A, 357, 1123

Elstner, D., Meinel, R., \& Rüdiger, G. 1990, Geophys. Astrophys. Fluid Dyn., 50, 85

Hawley, J. F., \& Balbus, S. A. 1991, ApJ, 376, 223

Hawley, J. F., Gammie, C. F., \& Balbus, S. A. 1995, ApJ, 440, 742

Hawley, J. F., Gammie, C. F., \& Balbus, S. A. 1996, ApJ, 464, 690

Kitchatinov, L. L., \& Mazur, M. V. 1997, A\&A, 324, 821

Kitchatinov, L. L., \& Rüdiger, G. 1997, MNRAS, 286, 757

Kley, W., Papaloizou, J. C. B., \& Lin, D. N. C. 1993, ApJ, 416,679

Koschmieder, E. L. 1993, Bénard Cells and Taylor Vortices (Cambridge University Press, Cambridge)

Matsumoto, L., \& Tajima, T. 1995, ApJ, 445, 767

Ogilvie, G. I., \& Pringle, J. E. 1996, MNRAS, 279, 152

Papaloizou, J. C. B., \& Terquem, C. 1997, MNRAS, 287, 771

Richard, D., \& Zahn, J.-P. 1999, A\&A, 347, 734

Ruzmaikin, A. A., Shukurov, A. M., \& Sokoloff, D. D. 1988, Magnetic fields of galaxies (Kluwer Academic Publishers, Dordrecht)

Rüdiger, G., \& Kitchatinov, L. L. 1996, ApJ, 466, 1078

Rüdiger, G., Primavera, L., Arlt, R., \& Elstner, D. 1999, MNRAS, 306, 913

Rüdiger, G., \& Drecker, A. 2001, Astron. Nachr., 322, 179

Ryu, D., \& Goodman, J. 1992, ApJ, 388, 438

Shakura, N. I., \& Sunyaev, R. A. 1973, A\&A, 24, 337

Stone, J. M., \& Norman, M. L. 1994, ApJ, 433, 746

Stone, J. M., \& Balbus, S. A. 1996, ApJ, 464, 364

Taylor, G. I. 1936, Proc. Roy. Soc. London A, 157, 546

Terquem, C., \& Papaloizou, J. C. B. 1996, MNRAS, 279, 767

Velikhov, E. P. 1959, Sov. Phys. JETP, 9, 995

Wendt, F. 1933, Ing. Arch., 4, 577 